Reinhard Heun ${ }^{1 *}$

\title{
How to write a scientific paper: A hypothesis-based approach
}

\author{
'University of Bonn, Germany \\ *email: globalpsychiatryagmx.com
}

DOI: 10.2478/gp-2018-0004

Received: 14 December 2017; Accepted: 20 December 2017

\begin{abstract}
Many books and other published recommendations provide a large, sometimes excessive amount of information to be included, and of mistakes to be avoided in research papers for academic journals. However, there is a lack of simple and clear recommendations on how to write such scientific articles. To make life easier for new authors, we propose a simple hypothesis-based approach, which consistently follows the study hypothesis, section by section throughout the manuscript: The introduction section should develop the study hypothesis, by introducing and explaining the relevant concepts, connecting these concepts and by stating the study hypotheses to be tested at the end. The material and methods section must describe the sample or material, the tools, instruments, procedures and analyses used to test the study hypothesis. The results section must describe the study sample, the data collected and the data analyses that lead to the confirmation or rejection of the hypothesis. The discussion must state if the study hypothesis has been confirmed or rejected, if the study result is comparable to, and compatible with other research. It should evaluate the reliability and validity of the study outcome, clarify the limitations of the study and explore the relevance of the supported or rejected hypothesis for clinical practice and future research. If needed, an abstract at the beginning of the manuscript, usually structured in objectives, material and methods, results and conclusions, should provide summaries in two to three sentences for each section. Acknowledgements, declarations of ethical approval, of informed consent by study subjects, of interests by authors and a reference list will be needed in most scientific journals.
\end{abstract}

\section{Keywords}

Psychiatry, neuroscience, publication, manuscript, hypothesis

\section{MANUSCRIPT CONTENT AND STRUCTURE}

Publication of new research is an essential step in scientific progress (Szabo et al. in press). Many books and other published recommendations provide a large, sometimes excessive amount of issues and information to be included, and of mistakes to be avoided in a scientific paper. However, there is a lack of simple and clear recommendations on how to write a manuscript. To make life easier for new authors, we propose a simple hypothesis-based approach, which consistently follows the study hypothesis, section by section, throughout the manuscript. For simplicity, we will refer to one study hypothesis in the following text, despite the fact that larger studies may intend to test several independent, connected or hierarchical hypotheses.

Scientific papers are usually structured in four sections, that is, introduction, material and methods, results and discussion. Other common parts of manuscripts are abstracts, the reference list and acknowledgements. Declarations of ethical approval by a registered committee, of informed consent and of interests of the authors are needed when investigating patient and control subjects.

Even though the headings and order may vary, the general approach is usually the same for most psychiatric and other medical journals. In simple terms, the introduction explains the scientific relevance and the contents of the study hypothesis. The methods section tells us how to test the hypothesis. The results section should provide the answer to testing the study hypothesis. The discussion should name and evaluate the outcome of testing of the study hypothesis in more general terms including its limitations and the potential implications on clinical practice or future research.

The following paragraphs will provide essential details on the roles and possible contents of the different sections using a hypothesis-based approach. 


\section{THE CONTENT OF THE ABSTRACT}

The abstract is usually put at the beginning of a paper. It should give two to three sentence summaries of each section of the manuscript, usually structured in objectives, material and methods, results and conclusions. The objectives introduce the concepts relevant for the study, develop and finally name the study hypothesis. The methods indicate the tools and the ways the hypothesis was tested. Results describe the data collection and analysis that lead to a confirmation or rejection of the hypothesis. Conclusions must indicate the confirmation or refusal of the hypothesis, validate the outcome, may explore the practical or theoretical relevance of the findings and may provide specific recommendations for necessary future research.

The importance of the abstract must not be underestimated as most researchers will decide if they are going to acquire and read the full paper based on the contents of the abstract.

\section{ROLE OF THE INTRODUCTION}

The introduction of the manuscript must develop and lead towards the study hypothesis, paragraph by paragraph. An initial paragraph may introduce the diseases or concepts to be investigated. If there are several diseases or concepts to be addressed, these could be explained in different paragraphs. Parts of such paragraphs or, if need be, individual paragraphs should introduce the general and specific relevance of the diseases or concepts to be investigated. Depending on the hypothesis to be developed, such relevance could be genetic, biological, clinical, therapeutic, societal, epidemiologic, financial, and so on.

If there are different concepts or issues to be investigated by the study, a later paragraph must explain the connections between the different aspects of the study questions. Assuming that most study hypotheses focus on the relevance of the possible interactions of different concepts, for example, diseases, prevalence, therapeutic approaches, and so on, there is a need to review and present the relevant literature covering and connecting these issues. This review of the relevant literature must summarize what is known about the relevant issues addressed by the study hypothesis, and what is unknown or unclear about these issues.

The final paragraph of the introduction should develop the hypothesis by indicating the missing information in the scientific literature, and what can be added to the scientific knowledge by testing the study hypothesis. In other words, what piece of the scientific puzzle can we get by testing the study hypothesis. This last paragraph of the introduction should finally explain and name the precise hypothesis in a way that allows the systematic testing of the study hypothesis. A precise description of the study hypothesis will introduce the following section on materials and methods, and will facilitate its understanding.

\section{ROLE OF MATERIAL AND METHODS}

The material and methods section may start by repeating the study hypothesis in more formal and technical terms, relating this to the different methods and conditions to be used for testing it. It may be useful to name the null/alternative hypothesis that is statistically tested.

It should be mentioned where and when the study was performed, and why these conditions have been chosen.

Next, the sample selection, the inclusion and exclusion criteria and the rationale for the choice of these must be described in a way that other researchers can understand these and would be able to reproduce these.

The choice of diagnostic instruments and interventions, and the rationale for these choices must be explained with the same precision. It will be important to define by whom, how and why the instruments and interventions were applied.

The outcome of the intervention or assessment must be defined and must lead to the explanation of how the outcome data have been collected, compiled, assessed and analysed to test the study hypothesis.

The statistical section or paragraph must clarify how the raw data were analysed to confirm or reject the hypothesis.

\section{PROPOSED CONTENTS OF THE RESULTS SECTION}

The results should start to give a precise description of the sample or material as it finally has been selected. Depending on the nature of the study hypothesis, the amount and necessary precision of demographic or material description may vary.

Further paragraphs should describe the results of interventions and assessments step by step following the course of the procedures outlined in the methods. 
The main results should be focussing on the evaluation of the study hypothesis and must provide a clear answer if the study hypothesis has been confirmed or rejected.

In case there are important and relevant results that have not been part of the initial intention of testing the study hypothesis, such results may be explained and summarised under the headings of results of explorative data analyses.

Tables and figures should be used within the results section if they can provide additional information or when study results can be presented more clearly or more economically than in text format. Table and figures should add to but not repeat the text, and vice versa.

\section{THE DISCUSSION SECTION}

The discussion section should initially state the confirmation or rejection of the hypothesis in more general and less technical terms than in the results section.

Following paragraphs should assess if the outcome of the study is in agreement and can be validated by similar studies and by supporting evidence from comparable studies within the research context of the hypothesis. Does the outcome of the study match or contradict other comparable publications, and if so, then why?

Another paragraph may explain if the outcome of the confirmation or refusal of the study hypothesis fits into the context of the relevant research covering the study question or if there are any contradictions, and if so, what the possible reasons for such inconsistencies may be.

Another essential paragraph may focus on the limitations of the study, the data collection, the data analyses, and on the limitations of the conclusion that can be made from the acceptance or rejection of the study hypothesis. In other words, how far can the results be generalised.

A conclusions paragraph may focus on what the outcome of the hypothesis testing means in particular and in general. How can this confirmation or rejection of the study hypothesis be used for clinical practice or future research, that is, for the testing of the next hypotheses?

\section{THE RELEVANCE OF ACKNOWLEDGEMENTS}

Acknowledgements should be added when other persons have substantially contributed or helped with different aspects of the paper such as providing material or helping with the literature search but do not qualify for full authorship. Authorship would indicate that authors have contributed in all aspects and stages of the study, and can claim scientific responsibility and can support its scientific validity.

\section{ROLE OF THE REFERENCES}

The reference list should be compiled in line with the authors' instructions of the journal for which the publication is intended. Literature that is relevant, necessary and sufficient to understand the development, testing, outcome and discussion of the study hypothesis must be cited, as long as it is not a part of the general scientific knowledge and/or not a part of the authors' personal knowledge or conclusions.

\section{LIMITATIONS OF THIS HYPOTHESIS-BASED PROPOSAL}

This proposal for a hypothesis-based writing of a scientific manuscript is formulated as a result of the experience of the author as reader, author, reviewer and editor of psychiatric and neurological papers. These recommendations are meant to help new researchers who are struggling with their first papers. They cannot and do not intend to be exhaustive. Covering all necessities, possibilities, complexities and potential mistakes of scientific articles would defeat the initial purpose of providing some simple and manageable guidance in a highly complicated scientific framework.

There are much more extensive recommendations and checklists to be found for different types of papers including the recommendations for systematic reviews such as PRISMA (Liberati et al 2009) and AMSTAR (Kung et al 2010) or for guidance papers (Heun and Gaebel 2015). Such recommendations may be helpful to check the format and completeness of the relevant information for specific types of papers. However, we are confident that using a hypothesis-based approach in all parts of a manuscript may be a reasonable and helpful aide-memoire and guidance for writing a scientific paper. Using the current hypothesis-based approach is unlikely to reduce the chances of acceptance and publication of a research article in a medical journal. 


\section{REFERENCES}

Heun R, Gaebel W. The relevance of EPA guidance papers in the framework of the European Psychiatric Association. European Psychiatry 30 (2015) 357-359

Kung J, Chiappelli F, Cajulis 00, Avezova R, Kossan G, Chew L, et al. Systematic Reviews to Clinical Recommendations for EvidenceBased Health Care: Validation of Revised Assessment of Multiple Systematic Reviews (RAMSTAR) for Grading of Clinical Relevance. Open Dent J 2010;4:84-91.

Liberati A, Altman DG, Tetzlaff J, Mulrow C, Gøtzsche PC, loannidis $J P$, et al. The PRISMA statement for reporting systematic reviews and meta-analyses of studies that evaluate health care interventions: explanation and elaboration. J Clin Epidemiol 2009;62(10):e1-34.

Szabo CP, Castaldelli-Maia JM; Chandra P, Cia A, Heun R, LecicTosevski D, Riba M, Tyrer P. Scientific publishing: a developmental role for the World Psychiatric Association. Global Psychiatry lin press) 